\section{Bacteria Use Type IV Pili to Walk Upright and Detach from Surfaces}

\author{
Maxsim L. Gibiansky, ${ }^{1 *}$ ]acinta C. Conrad, ${ }^{2 *}$ Fan Jin, ${ }^{1}$ Vernita D. Gordon, ${ }^{1}$ \\ Dominick A. Motto, ${ }^{4}$ Margie A. Mathewson, ${ }^{3}$ Wiktor G. Stopka, ${ }^{3}$ Daria C. Zelasko, ${ }^{3}$ \\ Joshua D. Shrout, ${ }^{4}$ Gerard C. L. Wong ${ }^{1,3} \dagger$
}

$\mathrm{B}$ acterial biofilms are multicellular surfacebound communities with important human health impacts (1). For Pseudomonas aeruginos $a$, a biofilm-forming pathogen responsible for lethal infections in cystic fibrosis (2), the motility appendages type IV pili (TFP) mediate the "twitching" motility mode observed in biofilms (3). Comparatively little is known about the transition from planktonic to surface-associated states that initiates biofilm formation. Here, we converted microscopy movies into searchable databases of bacterial behavior by using particletracking algorithms for quantitative analysis at fast time scales of all visible bacterial trajectories (4).

Shortly after attachment, before microcolony formation, we observed two TFP-driven surface motility mechanisms. Flagella-deficient $\Delta f l i M \mathrm{mu}-$ tants, whose movement is strictly TFP-dependent, "crawled" along their body axis with high directional persistence when oriented horizontally, parallel to the surface, and "walked" omnidirectionally with low directional persistence when attached vertically by one end (Fig. 1, A and B). Bacteria reversibly transitioned between these mechanisms. By contrast, Myxococcus xanthus slowly jiggles vertically before transitioning to a horizontal orientation for lateral crawling (5).

Each mechanism confers advantages for surface exploration (6). From the ensemble-averaged mean-square displacement, we found that vertical walking bacteria moved nearly diffusively, whereas horizontal bacteria could be divided into crawling superdiffusive and surface-anchored subdiffusive subpopulations (fig. S1). The average persistence length, $L_{\mathrm{p}}$, over which trajectories appear straight was shorter for walking bacteria $(2 \mu \mathrm{m})$ than for crawling bacteria $(6 \mu \mathrm{m})$; the former was similar to extension distances of TFP (7), suggesting that walking was caused by pulls of splayed TFP. Walking bacteria exhibited a higher instantaneous velocity [mean $71 \pm 2 \mathrm{~nm} / \mathrm{s}$ (SEM) versus $41 \pm 2$ $\mathrm{nm} / \mathrm{s}$, but crawling bacteria moved further on long time scales because of the $L_{\mathrm{p}}$. Crawling enabled directional motion; walking enabled rapid local exploration. These trends were preserved in wildtype (WT) bacteria.

Bacterial orientation played a key role in life cycle events. In $99 \%$ of 214 WT division events, one daughter cell remained attached horizontally; the majority $(67 \%)$ of the other daughters left the

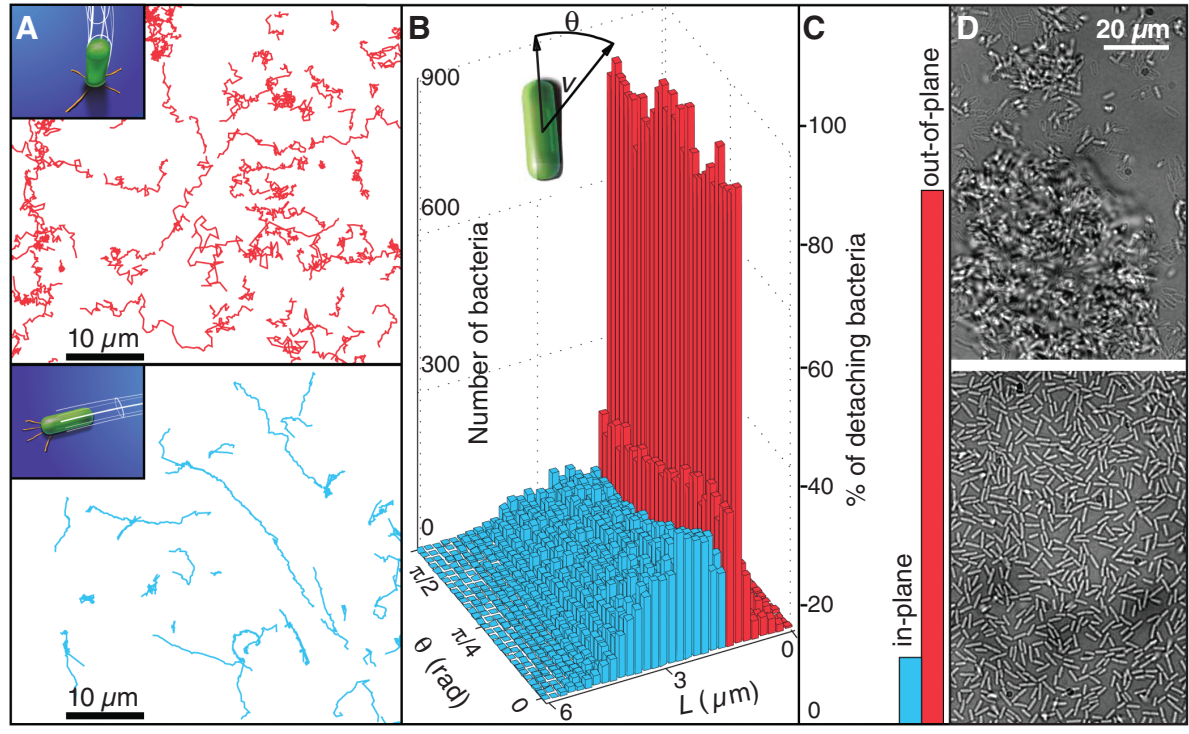

Fig. 1. TFP-mediated (A) vertical walking (top) and horizontal crawling (bottom) trajectories in $P$. aeruginosa. (B) Two-dimensional histogram of the angle between the bacterial axis and velocity $(\theta)$ and projected length ( $L$ ) of $N=70,073$ bacterial images showing populations of vertical (red) and horizontal (blue) bacteria. (C) Out-of-plane (vertical or tilted) orientations facilitate detachment. (D) $\triangle$ pilA biofilms had clusters (top); WT biofilms were uniform (bottom). division site by detaching, walking, or crawling. TFP governed this motility; TFP-deficient bacteria did not move apart after division. By using our search engine to locate all detachment events, we observed that detaching bacteria were overwhelmingly oriented out of plane (Fig. 1C). We found that TFP facilitated detachment by tilting from horizontal to vertical orientations; the influence of prevailing conditions was weak. TFPdeficient $\triangle$ pilA bacteria were defective in making this transition. This suggests a physical onset of biofilm formation, mediated by the transition from reversible polar attachment to irreversible longitudinal attachment (8).

Bacteria lacking TFP neither crawled nor achieved vertical orientations for walking and detachment. ApilA biofilms contained heterogeneous bacterial clusters (6) whose positions were determined by initial attachment sites (Fig. 1D). Divisions $\left(N_{\mathrm{d}}=79\right)$ outnumbered attachments $\left(N_{\mathrm{a}}=18\right)$ during 1 hour of cluster formation, indicating that clusters primarily grew via division. TFP-competent WT could actively walk, crawl, redistribute, and detach; despite a similar number of divisions $\left(N_{\mathrm{d}}=95\right)$, the WT biofilm did not contain clusters, indicating the $\triangle$ pilA biofilm morphology is caused by motility defects rather than adhesion defects.

References and Notes

1. J. W. Costerton, P. S. Stewart, E. P. Greenberg, Science 284, 1318 (1999).

2. T. Tolker-Nielsen et al., J. Bacteriol. 182, 6482 (2000).

3. J. S. Mattick, Annu. Rev. Microbiol. 56, 289 (2002)

4. Materials and methods are available as supporting material on Science Online.

5. H. Sun, D. R. Zusman, W. Shi, Curr. Biol. 10, 1143 (2000).

6. M. Klausen et al., Mol. Microbiol. 48, 1511 (2003)

7. J. M. Skerker, H. C. Berg, Proc. Natl. Acad. Sci. U.S.A. 98, 6901 (2001)

8. G. A. O'Toole, H. B. Kaplan, R. Kolter, Annu. Rev. Microbiol. 54, 49 (2000).

9. We thank S. Lee, G. O'Toole, T. Yahr, and M. Parsek for critically reading the manuscript. G.C.L.W. and co-workers are funded by the NIH, the NSF, and the Cystic Fibrosis Foundation.

\section{Supporting Online Material}

www.sciencemag.org/cgi/content/full/330/6001/197/DC1

Materials and Methods

Fig. S1

References

25 June 2010; accepted 2 August 2010

10.1126/science.1194238

${ }^{1}$ Department of Bioengineering, California Nano Systems Institute, University of California, Los Angeles, CA 90024, USA ${ }^{2}$ Department of Chemical and Biomolecular Engineering, University of Houston, Houston, TX 77204, USA. ${ }^{3}$ Department of Materials Science and Engineering, University of Illinois, UrbanaChampaign, IL 61801, USA. ${ }^{4}$ Department of Civil Engineering and Geological Sciences, University of Notre Dame, Notre Dame, IN 46556, USA.

*These authors contributed equally to this work.

†To whom correspondence should be addressed. E-mail: gclwong@seas.ucla.edu 


\section{Abstract}

Bacterial biofilms are structured multicellular communities involved in a broad range of infections. Knowing how free-swimming bacteria adapt their motility mechanisms near surfaces is crucial for understanding the transition between planktonic and biofilm phenotypes. By translating microscopy movies into searchable databases of bacterial behavior, we identified fundamental type IV pili-driven mechanisms for Pseudomonas aeruginosa surface motility involved in distinct foraging strategies. Bacteria stood upright and "walked" with trajectories optimized for two-dimensional surface exploration. Vertical orientation facilitated surface detachment and could influence biofilm morphology. 


\section{Science MIAAAS}

Bacteria Use Type IV Pili to Walk Upright and Detach from Surfaces

Maxsim L. Gibiansky, Jacinta C. Conrad, Fan Jin, Vernita D.

Gordon, Dominick A. Motto, Margie A. Mathewson, Wiktor G. Stopka, Daria C. Zelasko, Joshua D. Shrout and Gerard C. L. Wong (October 7, 2010)

Science 330 (6001), 197. [doi: 10.1126/science.1194238]

Editor's Summary

This copy is for your personal, non-commercial use only.

Article Tools Visit the online version of this article to access the personalization and article tools:

http://science.sciencemag.org/content/330/6001/197

Permissions Obtain information about reproducing this article:

http://www.sciencemag.org/about/permissions.dtl

Science (print ISSN 0036-8075; online ISSN 1095-9203) is published weekly, except the last week in December, by the American Association for the Advancement of Science, 1200 New York Avenue NW, Washington, DC 20005. Copyright 2016 by the American Association for the Advancement of Science; all rights reserved. The title Science is a registered trademark of AAAS. 